\title{
Elevated Cyclic Stretch Induces Aortic Valve Calcification in a Bone Morphogenic Protein-Dependent Manner
}

\author{
Kartik Balachandran, ${ }^{*}$ Philippe Sucosky, ${ }^{\dagger}$ \\ Hanjoong Jo, ${ }^{* \ddagger}$ and Ajit P. Yoganathan* \\ From the Wallace H. Coulter School of Biomedical Engineering," \\ and the Department of Cardiology, Emory University, Atlanta, \\ Georgia; and the Department of Mechanical and Aerospace \\ Engineering, ${ }^{\dagger}$ University of Notre Dame, Notre Dame, Indiana
}

Calcified aortic valve (AV) cusps have increased expression of bone morphogenic proteins (BMPs) and

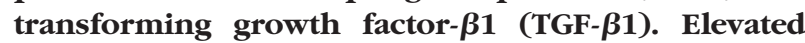
stretch loading on the $\mathrm{AV}$ is known to increase expression of matrix remodeling enzymes and pro-inflammatory proteins. Little, however, is known about the mechanism by which elevated stretch might induce AV calcification. We investigated the hypothesis that elevated stretch may cause valve calcification via a BMP-dependent mechanism. Porcine AV cusps were cultured in a stretch bioreactor, at $10 \%$ (physiological) or 15\% (pathological) stretch and 70 beats per minute for 3,7 , and 14 days, in osteogenic media supplemented with or without high phosphate (3.8 $\mathrm{mmol} / \mathrm{L})$, TGF- $\beta 1(1 \mathrm{ng} / \mathrm{ml})$, as well as the BMP inhibitor noggin $(1,10$, and $100 \mathrm{ng} / \mathrm{ml})$. Fresh cusps served as controls. Alizarin red and von Kossa staining demonstrated that $15 \%$ stretch elicited a stronger calcification response compared with $10 \%$ stretch in a fully osteogenic medium containing high phosphate and TGF- $\beta 1$. BMP-2, -4, and Runx2 expression was observed after 3 days on the fibrosa surface of the valve cusp and was stretch magnitude-dependent. Cellular apoptosis was highest at $15 \%$ stretch. Tissue calcium content and alkaline phosphatase activity were similarly stretchdependent and were significantly reduced by noggin in a dose dependent manner. These results underline the potential role of BMPs in valve calcification due to altered stretch. (Am J Pathol 2010, 177:49-57; DOI: 10.2353/ajpath.2010.090631)

Aortic valve calcification, long considered a passive process associated with risk factors such as age, hyperten- sion, renal disease, and cardiovascular disease, is potentially an active inflammatory and degenerative process that is influenced by hemodynamic forces as well as dysfunction of normal cellular functions. ${ }^{1-3}$ Surgical replacement or repair is the primary treatment mode for valve calcification as there are currently few medical or pharmacological options available. Calcified valves demonstrate increased expression of cytokines such as transforming growth factor- $\beta 1$ (TGF- $\beta 1$ ), 4,5 bone morphogenic proteins (BMPs), ${ }^{6}$ as well as alkaline phosphatase (ALP). ${ }^{7,8}$ Additionally, active osteogenesis was reported in calcified human valve cusps. ${ }^{1}$

Several mechanisms have been proposed for valve calcification including osteogenic differentiation of valvular interstitial cells, ${ }^{8,9}$ calcification secondary to cellular apoptosis, ${ }^{4,10}$ and necrosis-related deposition of calcium. ${ }^{11}$ Although a common feature of these processes is the disruption in the normal functioning of the endothelium and interstitial cells in the valve cusp, ${ }^{12,13}$ the exact mechanisms and factors that result in calcific disease initiation are not well understood. Apart from biochemical, atherogenic, and humoral factors, the mechanical/hemodynamic environment of the valve is thought to play an important role in the regulation of normal and pathological valve cell function. ${ }^{14,15}$

The aortic valve functions in a complex mechanical environment, which includes cyclic stretch, bending, pressure, and shear stresses, ${ }^{14}$ and several authors have linked alterations in these hemodynamic forces to initiation and progression of valve dysfunction and disease. ${ }^{16-18}$ Hypertension, which is a known risk factor for calcific aortic valve disease, ${ }^{19}$ results in a situation of elevated stress and stretch of the valve cusp. Under physiological conditions, the normal valve experiences

Supported by the National Science Foundation through the Engineering Research Center program at Georgia Institute of Technology under award number EEC-9731643.

Accepted for publication March 2, 2010.

Address reprint requests to Ajit P. Yoganathan, Ph.D., The Wallace H. Coulter School of Biomedical Engineering, Georgia Institute of Technology, 313 Ferst Dr, Suite 1121, Atlanta, GA 30332-0535. E-mail: ajit.yoganathan@bme.gatech.edu. 
approximately $10 \%$ stretch in the circumferential direction and $30 \%$ in the radial direction, which can change depending on changes in pressure load. ${ }^{20}$ Increased cyclic stretch has been linked to elevated expression of matrix remodeling proteins ${ }^{16}$ as well as pro-inflammatory markers ${ }^{21}$; however, the possible mechanisms of elevated stretch-induced valvular degeneration and specifically calcification are not well characterized.

We investigated the hypothesis that aortic valve calcification is induced by elevated cyclic stretch in a BMPdependent manner. The potential downstream effects of secreted BMP were blocked by using noggin. We found that the aortic valve can be calcified rapidly in a proosteogenic or atherogenic culture medium within 7 days, and that these responses were BMP-dependent.

\section{Materials and Methods}

\section{Tissue Harvest and Preparation}

Fresh porcine hearts (12 to 24 months) were obtained from a local abattoir within 10 minutes of slaughter. The aortic valves were transported to the laboratory in sterile, ice-cold Dulbecco's Phosphate Buffered Saline (Sigma, St. Louis, MO). A rectangular section of tissue with dimensions of $15 \times 10 \mathrm{~mm}$ was isolated from the central region of each valve cusp, proximal to the line of coaptation. Tissue sections were studied in circumferential orientation (Figure $1 \mathrm{~A}$ ) because this is the primary direction of the stress-bearing collagen fibers within the cusp. ${ }^{14}$ These samples were then randomized and assigned to treatment groups outlined below. We ensured that each cusp sample originated from a different animal and that there was a random mix of left, right, and noncoronary cusps for each treatment group. All samples were stretched in appropriate culture medium in a tensile stretch bioreactor (Figure $1 \mathrm{~B})^{22}$ maintained at $37^{\circ} \mathrm{C}$ in an incubator. Additionally, culture medium was replaced every 2 days during each experiment and was visually inspected for evidence of contamination. The waveforms imposed on the cusp are depicted in Figure 1C. ${ }^{20}$ On completion of the stretching, samples were removed and immediately washed three times in ice-cold sterile Dulbecco's Phosphate Buffered Saline and either frozen in optimal cutting temperature compound (Electron Microscopy Sciences, Hatfield, PA) for histological and immunohistochemical analyses, or flash frozen in liquid nitrogen.

\section{Experimental Groups and Conditions}

Three mechanical treatment groups were explored in this study: (1) fresh tissue (acute control); (2) 10\% stretch (physiological); and (3) 15\% stretch (hypertensive). Static controls were not used because they are not representative of a realistic mechanical environment for the aortic valve. We have validated previously that stretch is the dominant force acting on the cusp samples and that any shear stresses induced on the leaflet due to the stretching motion are negligible. ${ }^{22}$ Five different modifications of
A

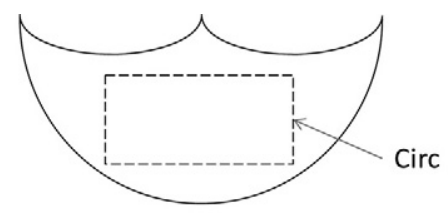

B

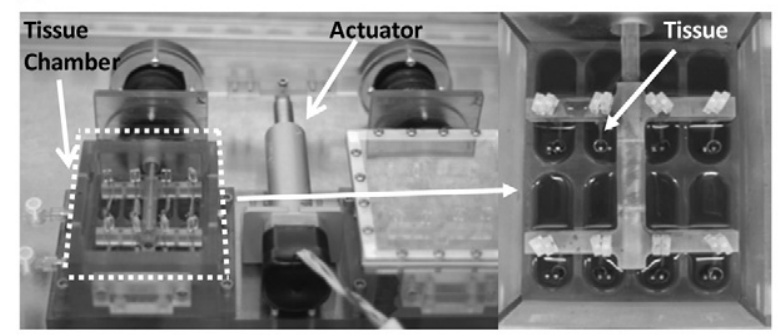

C Input waveforms to stretch bioreactor

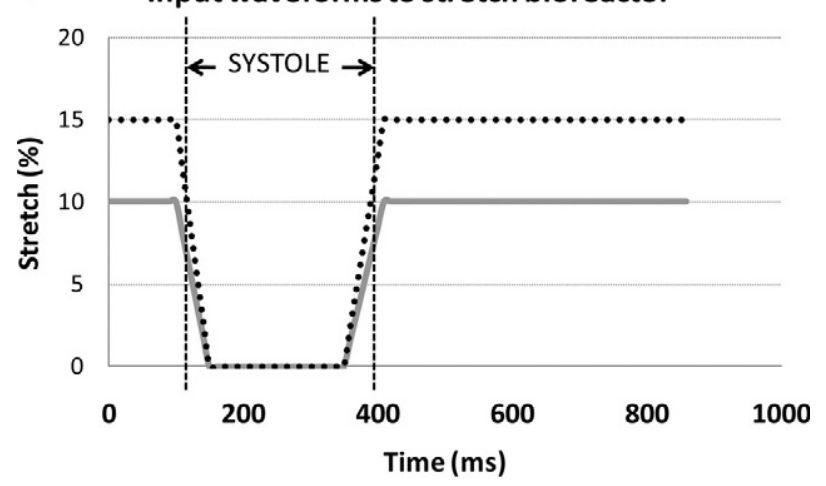

10\% Cyclic Stretch $\quad \cdots 115 \%$ Cyclic Stretch

Figure 1. Preparation of aortic valve cusps for the experiment. A circumferentially oriented $15 \times 10 \mathrm{~mm}$ section was excised from the central region of porcine aortic valve cusps (A). An ex vivo tensile stretch bioreactor was used in this study $(\mathbf{B}){ }^{22}$ A magnified image of the tissue chamber is shown on the right. A loading curve was used in this study. The gradients of the extension and relaxation approximated those experienced in vivo $(\mathbf{C}){ }^{20}$

Dulbecco's modified Eagle's medium (DMEM; Sigma) were initially used to understand their different pro-osteogenic and calcific potentials (Table 1). The high phosphate concentration $(3.8 \mathrm{mmol} / \mathrm{L})$ and inclusion of TGF- $\beta 1(1 \mathrm{ng} / \mathrm{ml})$ in the osteogenic medium were based on pathophysiologically relevant conditions reported in hyperphosphatemia due to renal disease ${ }^{23,24}$ and previous studies on cells in culture., ${ }^{4,25}$

To permit the characterization of the BMP-dependent pathway of aortic valve calcification, four different concentrations of the BMP-antagonist noggin (R\&D Systems, Minneapolis, MN), $-0,1,10$, and $100 \mathrm{ng} / \mathrm{ml}$, were added to the DMEM formulation that elicited the strongest calcific response (ie, DMEM formulation $V$ as shown in the results). Noggin was selected because it is a wellestablished BMP antagonist with a weak dissociation constant $\left(\mathrm{K}_{\mathrm{d}}\right)$ of 20 Picomolar. ${ }^{26}$ This would allow noggin to bind any expressed BMP and prevent its downstream action. Additionally, noggin has been previously shown to block the pro-inflammatory effects of BMP-4 expression on vascular endothelial cells. ${ }^{27}$ Therefore, it is an ideal candidate to evaluate the BMPdependence of aortic valve calcification. 
Table 1. Different Formulations of Culture Media Used in Order to Develop a Model for Calcification in an ex vivo System

\begin{tabular}{|c|c|c|}
\hline No. & Culture media & Composition \\
\hline 1 & Normal medium & Normal DMEM supplemented with $10 \%$ fetal bovine serum \\
\hline 2 & Base osteogenic medium & $\begin{array}{l}\text { Normal medium supplemented with } 1 \mathrm{mmol} / \mathrm{L} \beta \text {-glycerophosphate, } \\
10 \mu \mathrm{mol} / \mathrm{L} \text { dexamethasone, and } 0.9 \mathrm{mmo} / / \mathrm{PO}_{4}^{3-}\end{array}$ \\
\hline 3 & $\begin{array}{l}\text { Base osteogenic medium with high } \\
\text { phosphates }\end{array}$ & Base osteogenic medium with $3.8 \mathrm{mmol} / \mathrm{L} \mathrm{PO}_{4}{ }^{3-}$ \\
\hline 4 & Base osteogenic medium with TGF- $\beta 1$ & Base osteogenic medium with $1 \mathrm{ng} / \mathrm{ml}$ TGF- $\beta 1$ \\
\hline 5 & Full osteogenic medium & Base osteogenic medium with $3.8 \mathrm{mmol} / \mathrm{L} \mathrm{PO}_{4}{ }^{3-}$ and $1 \mathrm{ng} / \mathrm{ml} \mathrm{TGF}-\beta 1$ \\
\hline
\end{tabular}

\section{Experimental Sterility, Tissue Structural Integrity, and Viability}

For these studies, the valve tissue was being cultured in an ex vivo environment for up to 14 days. It was therefore important to verify that the tissue was not damaged and that there was no contamination of the culture media. Routine H\&E staining was used to assess tissue structure. Apart from a qualitative assessment, mean tissue thickness was determined from these H\&E micrographs by using the Image J software ( $\mathrm{NIH}$, Bethesda, MD). Tissue culture medium was analyzed microscopically every 2 days for evidence of bacterial or fungal growth. In addition, $\mathrm{pH}$ and media absorbance (at $364 \mathrm{~nm})^{18}$ was tested at 3, 7, and 14 days and compared with fresh media, as well as media conditioned for the same duration but without tissue. Cell death was determined by carefully examining 4',6-diamidino-2-phenylindole stained cells after 3,7 , and 14 days of stretch culture under a $40 \times$ objective. Individual cells were inspected for evidence of DNA aggregation, smearing, or other abnormalities. The count of these cells was then normalized over the total number of cells in that particular micrograph and presented as percentage cell death for that particular culture duration.

\section{Analysis of Calcification, Cell Apoptosis, and Bone Morphogenic Protein Expression}

Frozen optimal cutting temperature blocks were cut into $5-\mu \mathrm{m}$ sections in cross section. Alizarin Red and von Kossa staining were used to assay for tissue mineralization. Cell apoptosis was analyzed and quantified by using a terminal deoxynucleotidyl transferase-mediated dUTP nick-end labeling fluorescence kit (Roche Applied Science, Indianapolis, IN) as outlined before. ${ }^{16}$ Immunohistochemistry was used to identify cells positive for BMP-2 (1:25; Santa Cruz Biotechnology, Santa Cruz, CA) and BMP-4 (1:50; Santa Cruz Biotechnology). Semiquantitative assessments of BMP-2 and -4 expression were performed by using previously published methods. ${ }^{28} \mathrm{Sec}$ tions were also probed with von Willebrand Factor (1:100, Sigma) to identify the phenotype of the cells that were expressing BMPs.

\section{Calcium Assay}

Tissue samples were weighed and pulverized by using a mortar and pestle in liquid nitrogen, incubated in $1 \mathrm{M}$ acetic acid for 24 hours at $4^{\circ} \mathrm{C}$ to solubilize calcium, and centrifuged at $15,000 \mathrm{rpm}$. The supernatant as well as calcium standards were assayed for calcium content by using a calcium specific arsenazo dye reagent (FisherScientific, Waltham, MA). The quantity of reaction product formed was determined by spectrophotometry at $650 \mathrm{~nm}$. The calcium content was normalized with the weight of the sample and expressed as microgram calcium per milligram wet weight.

\section{Alkaline Phosphatase Enzyme Activity Assay}

Tissue samples were pulverized by using a mortar and pestle in liquid nitrogen and homogenized in ice-cold radioimmunoprecipitation assay buffer (Santa Cruz Biotechnology) before centrifuging at $15,000 \mathrm{rpm}$ to pellet extracellular matrix debris. The supernatant was assayed for protein concentration by using the BCA protein assay (Pierce, Rockford, IL). ALP activity in the tissue lysate was assayed by adding $10 \mathrm{mmol} / \mathrm{L} \mathrm{p}$ nitrophenyl phosphate as a substrate in $0.1 \mathrm{M}$ glycine buffer ( $\mathrm{pH}$ 10.4) containing $1 \mathrm{mmol} / \mathrm{L} \mathrm{ZnCl}_{2}$ and 1 $\mathrm{mmol} / \mathrm{L} \mathrm{MgCl}_{2}$. The quantity of $\mathrm{p}$-nitrophenol formed was determined by spectrophotometry at $405 \mathrm{~nm}$ and then monitored at 15,30,60,120, and 180 minutes and after 24 hours. ALP activity was calculated from a standard and normalized with the protein concentration. The specific activity of ALP was reported as nanomole per minute per milligram protein.

\section{Analysis of Downstream Transcription Factors for Bone Morphogenic Proteins}

Immunoblotting was used to analyze expression of Runx2 (1:100; Santa Cruz Biotechnology), Osteocalcin (1:50; Millipore, Billerica, MA), and $\beta$-actin (1:1000, Sigma). Equal amounts $(30 \mu \mathrm{g})$ of tissue lysates were resolved by reducing SDS-polyacrylamide gel electrophoresis. After transfer to a polyvinylidene difluoride membrane (Millipore), the blots were blocked with $3 \%$ bovine serum albumin and probed with primary antibody and appropriate biotinylated secondary antibody. The membranes were finally incubated in horseradish peroxidase-conjugated streptavidin. Immunopositive bands were detected by using a luminol-based chemiluminescence reagent (Pierce) and analyzed by densitometry by using the Image $\mathrm{J}$ program $(\mathrm{NIH})$. 


\section{Statistical Methods}

All quantitative data were expressed as mean \pm SE. The sample size was $n=6$ to 8 (all from different animals) for all analyses. The data were first analyzed by using analysis of variance to determine whether there was significant contribution by a particular treatment on the measured parameters, followed by the Tukey posthoc test. A $P$ value of less than 0.05 was used as a measure of statistical significance. All statistical analyses were performed by using Minitab (Minitab Inc., State College, PA).

\section{Results}

Tissue Structure, Viability, and Media Sterility is Maintained under Cyclic Stretch

H\&E staining (Figure 2A) demonstrated that the tissue morphology and structure was comparable between fresh valve cusps and cusps stretched for 3, 7, and 14 days. Tri-layered morphology was maintained, and characteristic features such as the ridged fibrosa layer and sparse spongiosa layer was maintained between the stretched groups and fresh tissue. In addition, tissue thickness was statistically $\operatorname{similar}(P>0.4 ; n=10)$ between fresh controls and cusp samples stretched for 3, 7, and 14 days (Figure 2B). Analysis of cell death showed that the number of dead cells was less than $3.5 \%$ of number of total cells (Figure 2, C and D), indicating that the bioreactor system did not induce significant cell death. The number of cells that appeared to be necrotic were statistically similar $(P>0.1)$ between 3,7 , and 14 days of stretch culture.

There was no significant $(P>0.5 ; n=6)$ difference in absorbance at $364 \mathrm{~nm}$ between fresh DMEM (0.408 \pm $0.025)$, conditioned medium with tissue after $3(0.432 \pm$ $0.035), 7(0.427 \pm 0.036)$, and 14 days $(0.421 \pm 0.031)$, and conditioned medium without tissue after 3 (0.408 \pm $0.025), 7$ (0.415 \pm 0.029$)$, and 14 days (0.420 \pm 0.029$)$. Media $\mathrm{pH}$ was also statistically unchanged $(P>0.5 ; n=$ 12) over the course of experiment, indicating good media sterility. In addition, examination of the media under the microscope every 2 days did not demonstrate evidence of bacterial or fungal contamination.

\section{Cyclic Stretch Induced Aortic Valve Calcification}

It has not been previously demonstrated whether aortic valve cusp tissue can be calcified ex vivo in an experimentally feasible timeframe. We tested the hypothesis that porcine aortic valve cusps can be calcified by a combination of pathological levels of stretch (15\%) and a pathophysiologically relevant humoral environment (high concentrations of phosphate and TGF- $\beta 1$ ). Fresh porcine valve samples and samples cultured for 7 days at $10 \%$ stretch (physiological) or $15 \%$ stretch (pathological) in normal DMEM (Figure 3, A and E), osteogenic medium (Figure 3, B and F), or osteogenic medium supplemented with high phosphate (Figure 3, C and $\mathrm{G}$ ) did not show positive Alizarin red staining for calcification. Calcific

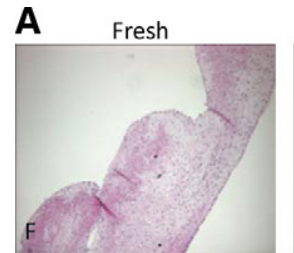

7 days

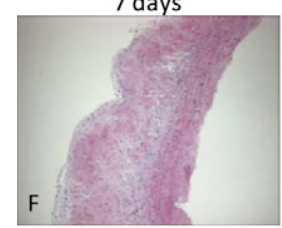

B
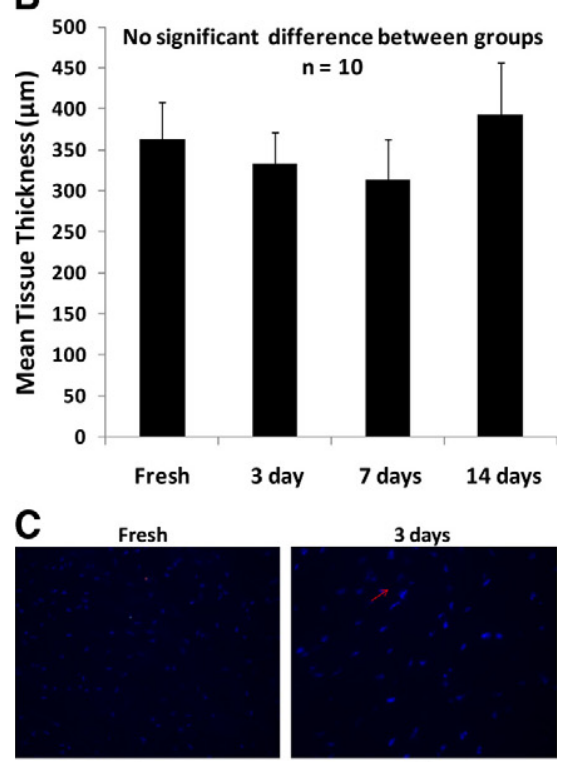

7 days
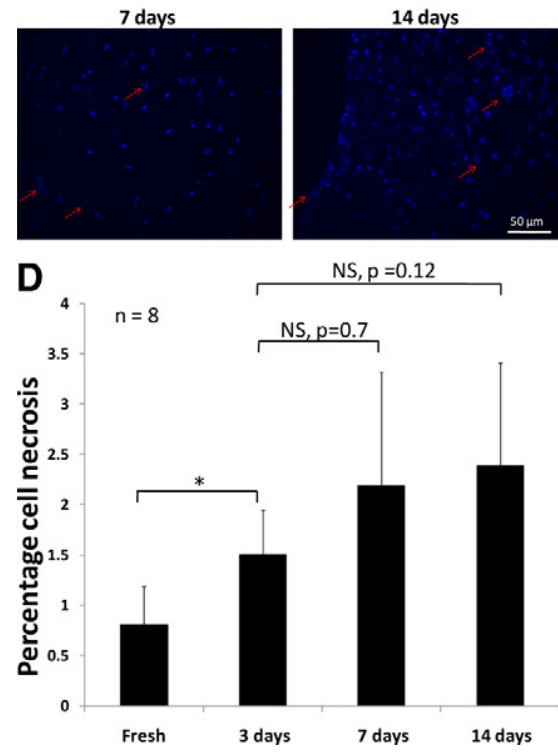

Figure 2. Normal tri-layered morphology of aortic valve cusps was maintained in stretch experiments. Tissue morphology and structure was comparable between fresh controls and samples stretched for 3, 7, and 14 days (A). There was no significant difference in tissue thickness between fresh controls and samples stretched for 3, 7, and 14 days (B) $[n=10]$. Samples cultured to 3,7 , and 14 days showed some evidence of DNA deterioration as demonstrated by arrows $(\mathbf{C})$; however, the number of necrotic cells was less than $3.5 \%$ of the total number of cells $(\mathbf{D})[n=8] \mathrm{F}$ indicates fibrosa. ${ }^{*} P<0.05$. 

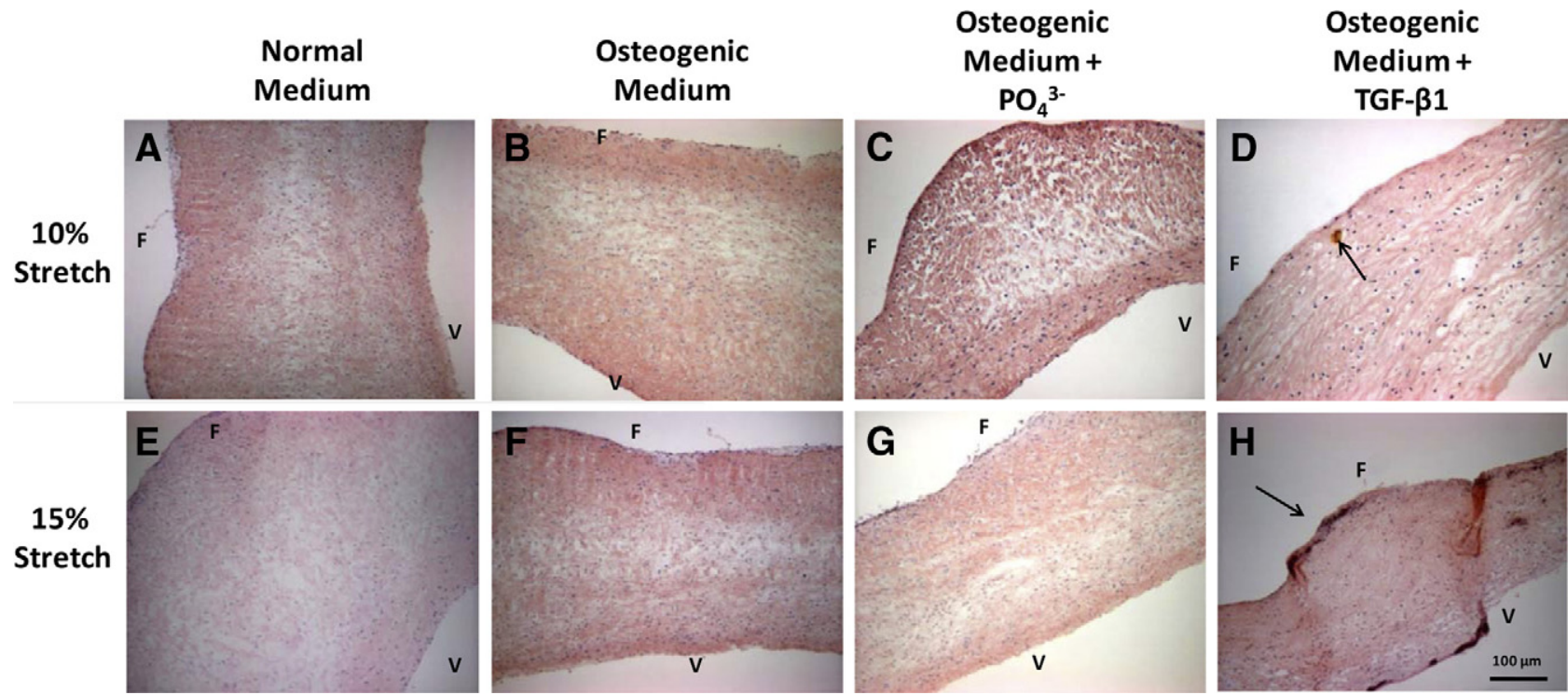

Figure 3. Alizarin red staining of porcine aortic valve cusps that were cultured for seven days under $10 \%(\mathbf{A}-\mathbf{D})$ or $15 \%(\mathbf{E}-\mathbf{H})$ stretch in normal DMEM, osteogenic DMEM, osteogenic DMEM with high phosphate $(3.8 \mathrm{mmol} / \mathrm{L})$, and osteogenic DMEM with $1 \mathrm{ng} / \mathrm{ml}$ TGF- $\beta 1$, as indicated. Small calcific nodules were found only in $\mathbf{G}$ and $\mathbf{H}$ as marked with arrows. $\mathrm{F}$ indicates fibrosa; $\mathrm{V}$, ventricularis.

nodule formation could be observed on the aortic surface in cusp samples exposed to both $10 \%$ and $15 \%$ stretch in osteogenic media with TGF- $\beta 1$ (Figure 3, D and H).

Treatment of valve cusps in fully osteogenic medium under 10\% (physiological) or 15\% stretch (pathological) for 7 days resulted in calcification in a stretch-dependent manner (Alizarin Red, Figure 4, A and C; von Kossa, Figure 4, B and D). The fibrosa side showed more intense staining than the ventricularis side of the cusp when exposed to $15 \%$ stretch. These results suggest that calcification of aortic valve cusps required both stretch and the fully osteogenic medium containing high phosphate and TGF- $\beta 1$ levels, whereas neither stretch nor the fully osteogenic medium alone was sufficient. In addition, the fibrosa side appeared to be preferentially susceptible to calcification.
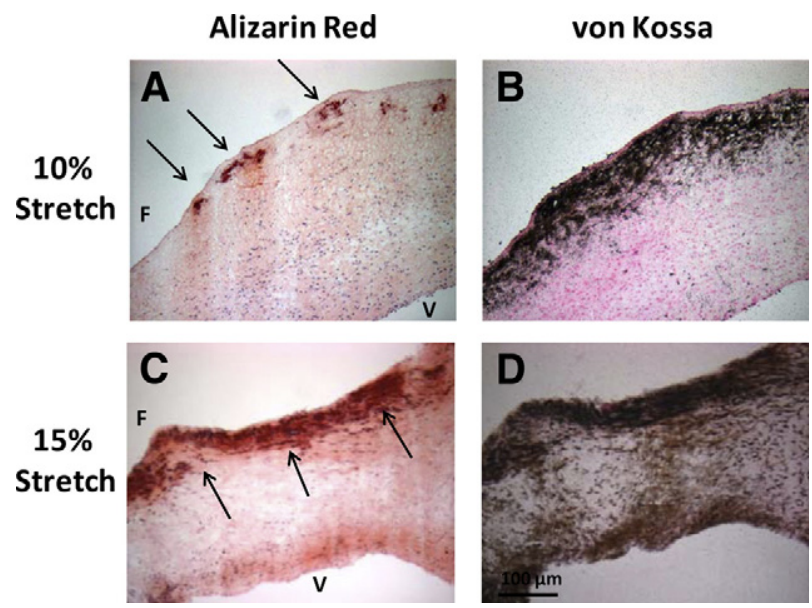

Figure 4. Cyclic stretch induced calcification of porcine aortic valve cusps. Aortic valve cusps were exposed to $10 \%$ (A and $\mathbf{B}$ ) or 15\% (C and D) cyclic stretch for seven days in fully osteogenic medium containing both high phosphate and TGF- $\beta 1$. Cusps were then stained with Alizarin Red (A and $\mathbf{C}$ ) or Von Kossa (B and D). Calcific nodules are marked with arrows. F indicates fibrosa; V, ventricularis.

\section{Cellular Apoptosis was Highest for the 15\% Stretch and Lowest for 10\% Stretch}

Because previous authors have linked valve calcification to cellular apoptosis, ${ }^{4}$ we tested if exposing cells to physiological and/or pathological stretch for 7 days in the fully osteogenic medium induced apoptosis of aortic valve cusp cells. Terminal deoxynucleotidyl transferase-mediated dUTP nick-end labeling assay (Figure 5, A and B) showed significantly larger numbers of apoptotic cells when cusps were cultured with 15\% stretch (180.6 \pm 49.5 ) in the fully osteogenic medium compared with $10 \%$ stretch (22.4 \pm 4.3) and fresh controls (4.2 \pm 0.9$)$. In addition, cellular apoptosis was significantly reduced when samples were cultured with $100 \mathrm{ng} / \mathrm{ml}$ noggin $(10 \%$ stretch: $3.2 \pm 0.9 ; 15 \%$ stretch: $12.7 \pm 1.9)$. For the $10 \%$ stretch with noggin group, the number of apoptotic cells was statistically similar to fresh controls.

\section{Pathological Stretch Increased BMP-2/-4 Expression in Aortic Valve Cusps}

To determine whether BMP expression was increased by cyclic stretch in the fully osteogenic medium, BMP-2 and -4 expressions were examined in aortic cusps that were stretched to $10 \%$ and $15 \%$ and in fresh controls (Figure $6)$. Fresh samples did not show any discernable amount of BMP-2 or BMP-4 as detected by immunohistochemistry. Both $10 \%$ and $15 \%$ cyclic stretch significantly increased BMP-2 and -4 expressions. In addition, BMP expression was significantly higher at $15 \%$ stretch compared with 10\% stretch (Figure 6B). BMP-2 and BMP-4 expression was remarkably higher on the fibrosa side of the cusps compared with the ventricularis (Figure 6A). In addition, double immunostaining on the same valve sample with BMP-2 and von Willebrand Factor (Figure 
A
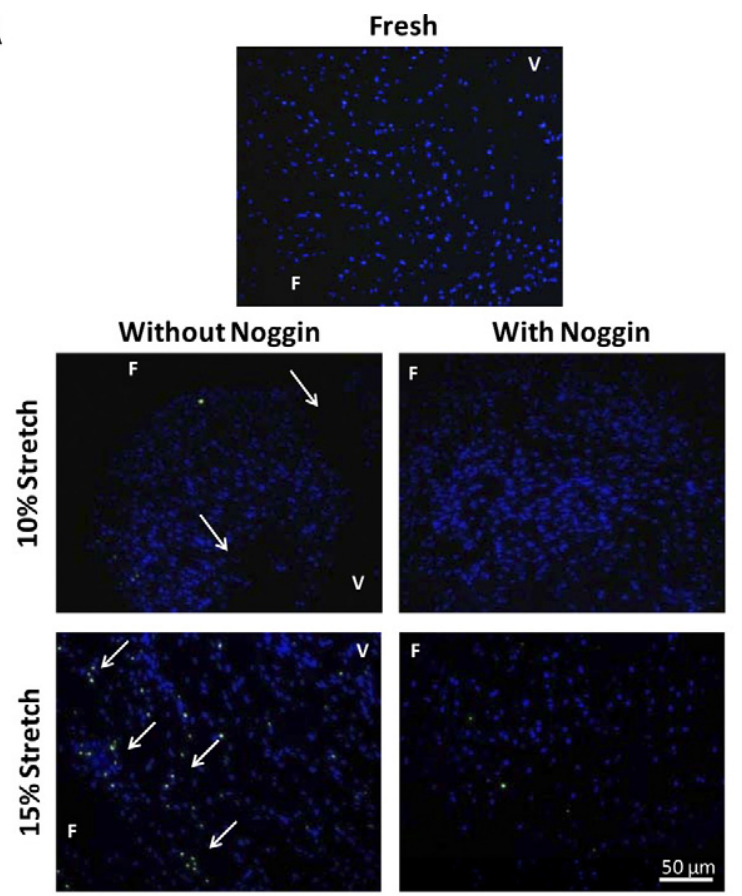

B
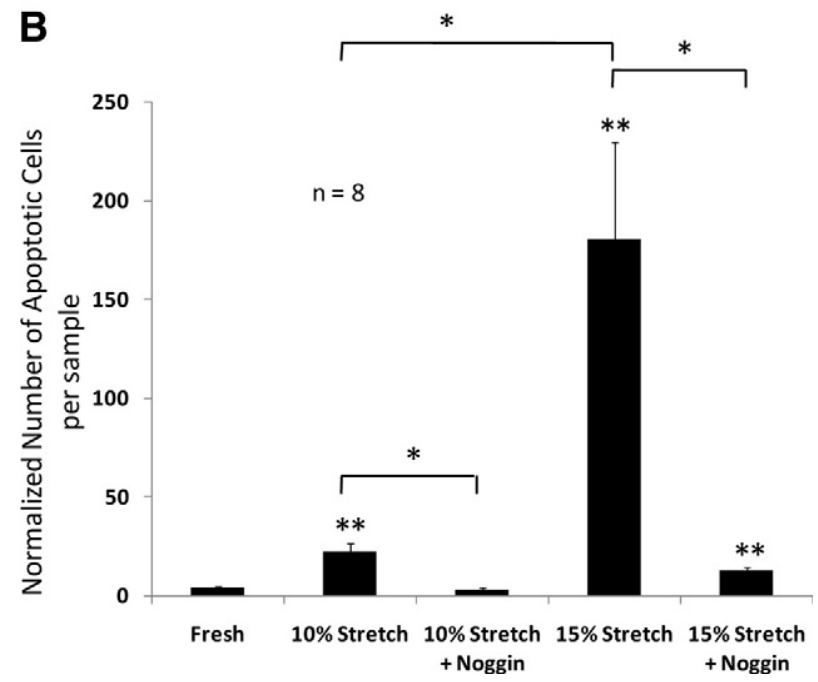

Figure 5. Elevated stretch-induced apoptosis of porcine aortic valve cusp cells. Fresh valve cusps and cusps exposed to $10 \%$ or $15 \%$ cyclic stretching in fully osteogenic medium were assayed for apoptosis by terminal deoxynucleotidyl transferase-mediated dUTP nick-end labeling staining (green) with 4',6-diamidino-2-phenylindole nuclei counterstain (blue), with apoptotic cells detected marked with arrows (A). The normalized number of apoptotic cells per image field was then quantified for each treatment condition (B). F indicates fibrosa; $\mathrm{V}$, ventricularis. ${ }^{*} P<0.05 ;{ }^{* * *} P<0.05$ vs. fresh control.

7, A-D) revealed strong colocalization of these two proteins (Figure 7).

\section{Calcification of Aortic Valve Cusps Occur in a BMP-Dependent Manner}

Next, we examined whether cusp calcification induced by pathological cyclic stretch was mediated in a BMPdependent manner. To test this hypothesis, cusps were exposed to $10 \%$ or $15 \%$ cyclic stretch for 14 days in the fully osteogenic medium supplemented with or without
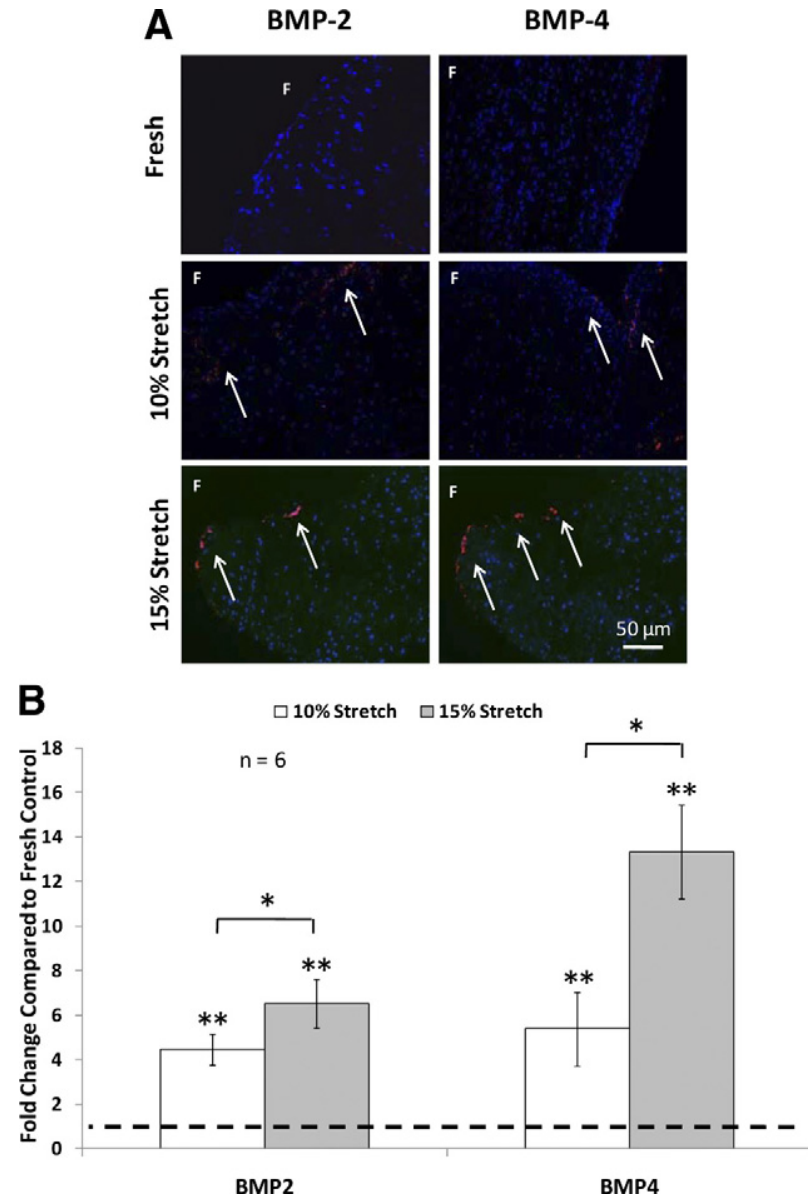

Figure 6. Representative micrographs of BMP-2 and BMP-4 immunohistochemical staining (A) and semiquantification (B) after three days of culture (10\% or $15 \%$ stretch) in fully osteogenic DMEM compared with fresh controls. Expression of BMP was primarily observed on the fibrosa surface of the valve cusp. Arrows represent immunopositive cells. $\mathrm{F}$ indicates fibrosa. ${ }^{*} P<0.05$; ${ }^{* * *} P<$ 0.05 vs. fresh control.

the BMP inhibitor noggin. Then, total calcium content in each cusp was determined by calcium arsenazo assay (Figure 8A). Exposure of cusps to pathological stretch $(15 \%)$ increased the calcium content by fivefold com-
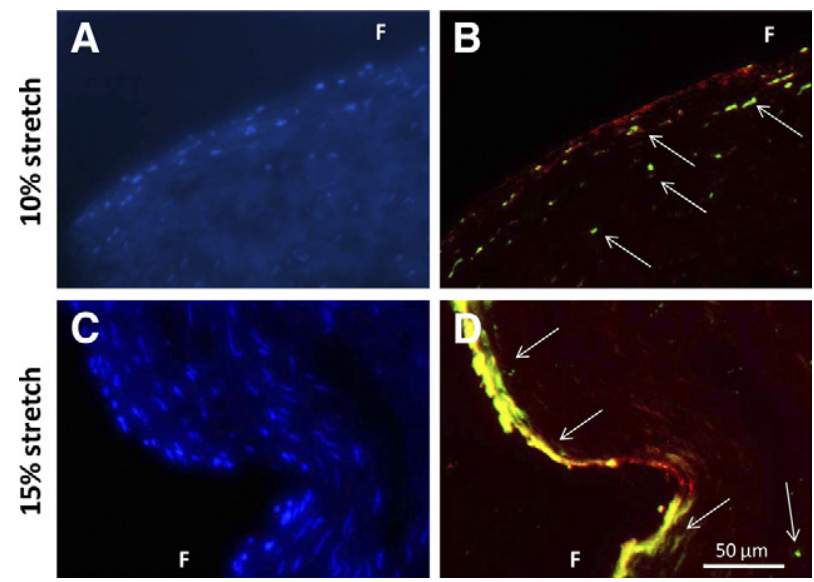

Figure 7. Representative micrographs of BMP-2 (green) and von Willebrand Factor (red) double immunohistochemical staining for samples stretched at $10 \%$ (A and $\mathbf{B})$ and $15 \%(\mathbf{C}$ and $\mathbf{D})$ in fully osteogenic media. Cell nuclei are stained with 4',6-diamidino-2-phenylindole (blue) and presented in a separate image for clarity of viewing. Arrows represent immunopositive cells. F indicates fibrosa. 

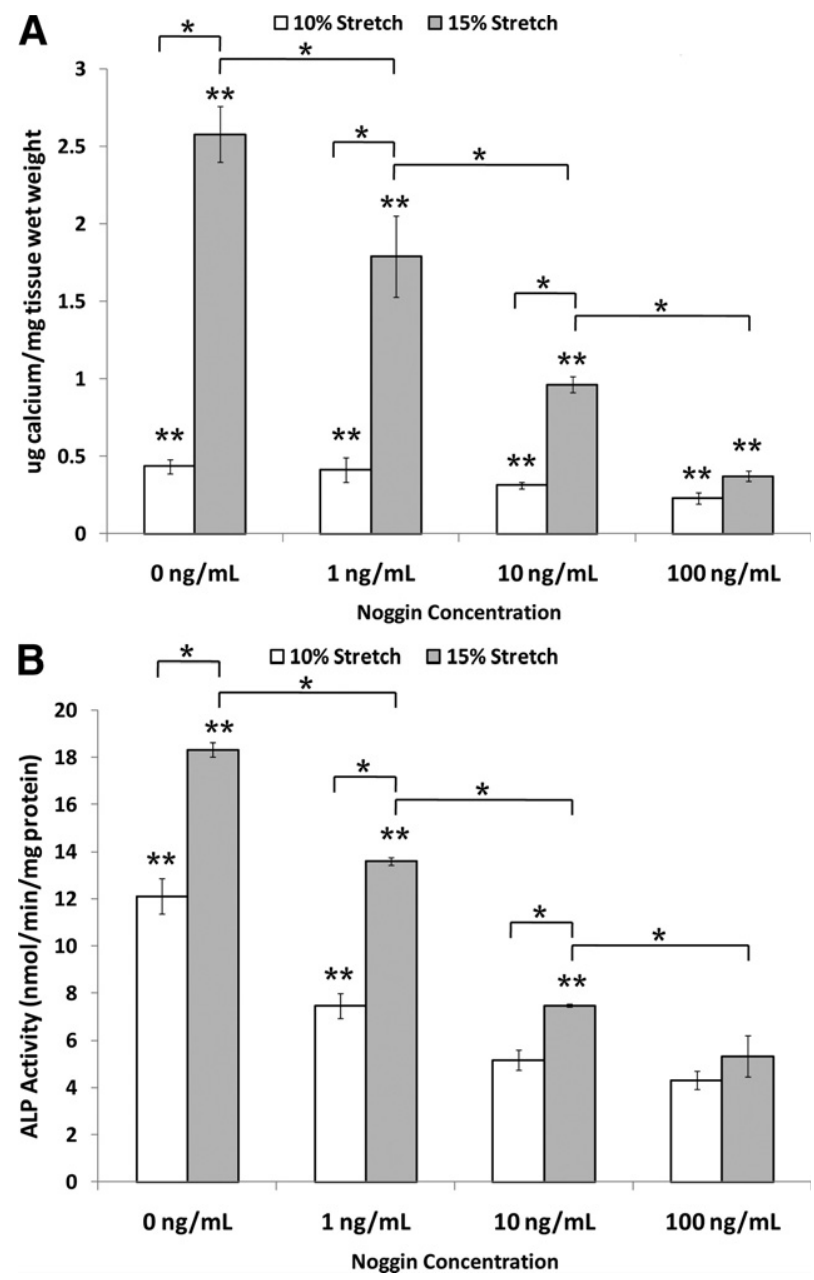

Figure 8. Calcium content in stretched (10\% and 15\%) valves cultured in fully osteogenic DMEM in $0,1,10$, and $100 \mathrm{ng} / \mathrm{ml}$ noggin for 14 days (A) $[n=$ 8]. ALP activity in stretched (10\% and $15 \%)$ valves cultured in fully osteogenic DMEM in $0,1,10$, and $100 \mathrm{ng} / \mathrm{ml}$ noggin for seven days $(\mathbf{B})[n=8] .{ }^{*} P<0.05$; *** $P<0.05$ versus fresh control.

pared with normal stretch (10\%). Moreover, noggin blocked stretch-induced calcium increase in a concentration dependent manner (Figure 8A). As low as $1 \mathrm{ng} / \mathrm{ml}$ of noggin showed a significant inhibition $(P<0.05)$, reaching a maximum inhibition level at $100 \mathrm{ng} / \mathrm{ml}$.

We next examined ALP activity in cusps exposed to $10 \%$ or $15 \%$ cyclic stretch for 7 days in the fully osteogenic medium. ALP activity in fresh cusp tissue was $1.7 \pm 0.8 \mathrm{nmol} / \mathrm{min} / \mathrm{mg}$ protein. Fifteen percent stretch significantly increased ALP activity above that of $10 \%$ stretch at all concentrations of noggin (Figure 8B). Importantly, noggin treatment inhibited stretch-induced ALP activity in a concentration-dependent manner. These results suggest that calcification induced by a pathological level of stretch is mediated in a BMP-dependent manner.

Runx2 is a key transcription factor associated with osteoblast differentiation and is also a downstream transcription factor for BMP-2 and -4. Immunoblotting studies (Figure 9) demonstrated that Runx2 expression (Figure 9A) of aortic cusps was significantly increased by 7 days of $15 \%$ stretch in the fully osteogenic medium, in comparison with fresh controls (2.24-fold) and $10 \%$ stretch
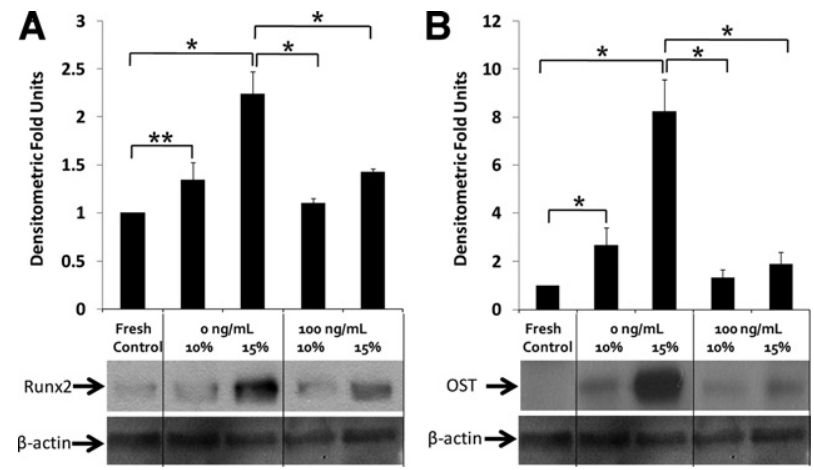

Figure 9. Runx2 expression in stretched (10\% and 15\%) valves cultured in fully osteogenic DMEM in 0 and $100 \mathrm{ng} / \mathrm{ml}$ noggin for three days (A) $[n=6]$. Osteocalcin (OST) expression in stretched (10\% and $15 \%)$ valves cultured in fully osteogenic DMEM in 0 and $100 \mathrm{ng} / \mathrm{ml}$ noggin for seven days $(\mathbf{B})[n=6] .{ }^{*} P<0.05 ;{ }^{* *} P<0.01$

(1.67-fold). One hundred nanograms per milliliter noggin treatment inhibited stretch-induced expression of Runx2. The importance of stretch is also emphasized by the fact that there was no significant difference between fresh controls and normal (10\%) stretch groups.

Identical trends were observed for osteocalcin expression (Figure 9B). Osteocalcin expression was significantly increased by both 10\% (2.67-fold) and 15\% (8.25fold) stretch when compared with fresh controls. These fold changes were greater than those observed for Runx2, which may be attributed to low basal levels of osteocalcin in a native aortic valve. In the presence of $100 \mathrm{ng} / \mathrm{ml}$ noggin, there was significant reduction in osteocalcin expression for both 10\% (0.50-fold) and 15\% (0.23-fold) stretched groups.

\section{Discussion}

The novel contributions of this study are the following: (1) we have demonstrated that the aortic valve can be calcified preferentially on the fibrosa side within a week in response to a pathologically relevant level of cyclic stretch and pro-osteogenic humoral conditions in ex vivo tissue culture; (2) pathological stretch induced apoptosis and BMP expression preferentially on the fibrosa side; and (3) the BMP antagonist noggin was able to block osteogenic and calcification events (ALP activity, calcium content, Runx2, and osteocalcin expression in the valve cusps/tissue) that were induced by stretch. This study therefore points to the BMPs, particularly BMP-2 and/or BMP-4 as key players in stretch-induced aortic valve calcification in a pro-osteogenic environment. These findings also suggest a potential importance of BMP antagonists in aortic valve calcification.

One of the most important findings of this current work is that aortic valve cusps can be calcified within a week by treating with a pathologically relevant level of stretch $(15 \%)$ in fully osteogenic medium containing both high phosphate content $(3.8 \mathrm{mmol} / \mathrm{L})$ and TGF- $\beta 1(1 \mathrm{ng} / \mathrm{ml})$. Neither the stretch nor the fully osteogenic medium alone could induce valve calcification within an experimental time frame of up to 14 days (data not shown). These 
results suggest that stretch and the fully osteogenic medium act in an additive manner. Although full mechanistic insights need additional studies, initial data suggest increased BMP-4 expression, cellular apoptosis, subsequent Runx2 and osteocalcin expression, and ALP activity as potential mechanisms. Reaching a threshold level of BMP by the additive actions of the mechanical (stretch) and atherogenic (TGF- $\beta 1$ and phosphate concentration) factors may be a key event in determining if the aortic valve undergoes calcification. A recent review article revealed that the specific atherogenic factors studied in the present study are especially important to the progression of valve calcification. ${ }^{15}$

The ex vivo tissue culture system used in this study is an accelerated model for calcification, obtaining expression of BMPs and ALP after 3 and 7 days, respectively, and significant increases in tissue calcium content after 14 days. It did not induce contamination and was able to maintain tissue viability for the experimental durations studied. An important contribution of this study is that this ex vivo system could be used to study valvular disease mechanisms on the bench-top before more detailed studies are done in vivo. Indeed, our studies may provide insight into possible mechanisms for stretch-induced/hypertension-induced valve disease. BMP-blockers appear to be a potential exciting target for future studies toward pharmacological treatment of aortic valve calcification.

It was observed that calcification and tissue expression of BMP was preferentially expressed on the fibrosa side of the valve. Although the reason for this occurrence is not clear, potential reasons could be the fact that the cells on the fibrosa side are genetically more prone to calcification, ${ }^{29}$ or that the local in vivo hemodynamic conditions on the aortic side of the valve cusps prime them to be more procalcific in nature. ${ }^{28}$ Additionally, most of the expression of BMPs was observed on the endothelial layer of the valve cusps (Figure 6). This is in agreement with previous results on the effects of pathological shear stresses on valve cusps. ${ }^{28}$ It is speculated that the endothelial layer acts as the mechanosensor in the case of cyclic stretch.

Other studies have also demonstrated that addition of BMP and TGF- $\beta 1$ can independently result in osteogenic differentiation of aortic valve cells cultured in vitro. ${ }^{4,8}$ BMP-2 specifically can induce calcific nodule formation of aortic valve fibroblasts. ${ }^{30}$ This study demonstrated that BMPs and TGFs are more closely related than previously thought, with addition of exogenous TGF- $\beta 1$ directly resulting in expression of BMP-2 and -4 after only 3 days. We reported recently that altered shear stresses result in aortic valve inflammation via a BMP-dependent pathway, ${ }^{28}$ indicating a strong link between altered mechanical forces on the valve tissue and BMP-influenced valve dysfunction. This study is also in agreement with in vitro studies on mesenchymal cells where BMP signaling activated the chondro-osteogenic Runx2/Cbfa pathway. ${ }^{15}$ Increased serum phosphate concentrations have also been linked to activation of the Runx2/Cbfa pathway, ${ }^{15}$ which was also observed in this study.

It is believed that calcification could occur via either an apoptosis-driven pathway ${ }^{10,11,31}$ or via an osteogenic and more matrix-dependent pathway. ${ }^{10,11}$ Some studies have also demonstrated that depending on the mechanical and biochemical environment both processes can potentially occur. ${ }^{4,11}$ These studies also demonstrated that TGF- $\beta 1$ stimulates cellular apoptosis. ${ }^{4,11}$ We demonstrated that cellular apoptosis was present for samples stretched to $15 \%$, but to a lesser extent in $10 \%$ stretch. Apoptotic cells were predominantly expressed on the fibrosa, matching the observed pattern of preferential calcification on that same surface of the valve. This finding further lends strength to the speculation that valve calcification is side-specific. In addition, ALP activity, which is a marker for osteogenic differentiation was also higher at 15\% stretch compared with 10\% stretch. It is believed that both apoptosis- and osteogenic-driven calcification could be occurring, but further study is needed to confirm this.

It was demonstrated that noggin can effectively inhibit BMP-induced aortic valve calcification. Noggin was used in this study as an antagonist for BMPs. Other BMP antagonists include chordin and follistatin. ${ }^{32}$ Although these other molecules were not investigated, noggin is well established as a specific BMP antagonist with a weak dissociation constant $\left(\mathrm{K}_{\mathrm{d}}\right)$ of $20 \mathrm{pM},{ }^{26}$ making it a good candidate for this study. Noggin has been shown to block the pro-inflammatory effects of BMP-4 expression on vascular endothelial cells. ${ }^{27}$ Indeed, the value of noggin in this study is its ability to interfere with the action of BMPs while not affecting the expression of BMPs per se. Ultimately, valve calcification could be a result of a breakdown of this delicate balance between agonists (BMPs) and antagonists (noggin) for calcific progression.

The importance of mechanical cues on tissues such as the aortic valve is clearly underlined in this study, both for normal homeostasis as well as the progression of pathology. Matrix remodeling enzymes, which are also involved in valve calcification, and investigated in a previous studies, were also seen to be modulated by altered cyclic stretch. ${ }^{16,33}$ From these results, it can be speculated that elevated cyclic stretch primes the aortic valve to an activated state, which is more prone to disease. Indeed, in this study, the presence of pro-osteogenic conditions readily induced progression of valve calcification. In addition, altered shear stress increased BMP-dependent inflammation on the fibrosa surface of aortic valve cusps. ${ }^{28}$ In a recent study, it was shown that matrix stiffness can modulate the differentiation of valve interstitial cells to a pathological phenotype. ${ }^{11}$

In conclusion, this study strongly suggests the importance of BMPs in aortic valve calcific progression resulting from elevated stretch. Moreover, the importance of studying valve pathology in close relation with its mechanical environment is also clearly demonstrated. Further studies should focus on understanding the detailed mechanobiological signaling of BMP-induced valve cell osteogenic differentiation.

\section{Acknowledgments}

We thank Dr. Michael S. Sacks (University of Pittsburgh) for the design of the stretch bioreactor. We thank Holifield 
Farms (Covington, GA) for donating the porcine hearts for this study.

\section{References}

1. Mohler ER, 3rd: Mechanisms of aortic valve calcification. Am J Cardiol 2004, 94:1396-1402, A1396

2. Otto CM, Kuusisto J, Reichenbach DD, Gown AM, O'Brien KD: Characterization of the early lesion of "degenerative" valvular aortic stenosis: histological and immunohistochemical studies. Circulation 1994, 90:844-853

3. Chester AH, Taylor PM: Molecular and functional characteristics of heart-valve interstitial cells. Philos Trans R Soc Lond B Biol Sci 2007, 362:1437-1443

4. Clark-Greuel JN, Connolly JM, Sorichillo E, Narula NR, Rapoport HS, Mohler ER, 3rd, Gorman JH, 3rd, Gorman RC, Levy RJ: Transforming growth factor-beta1 mechanisms in aortic valve calcification: increased alkaline phosphatase and related events. Ann Thorac Surg 2007, 83:946-953

5. Jian B, Narula N, Li QY, Mohler ER, 3rd, Levy RJ: Progression of aortic valve stenosis: TGF-beta1 is present in calcified aortic valve cusps and promotes aortic valve interstitial cell calcification via apoptosis. Ann Thorac Surg 2003, 75:457-465; discussion 465-466

6. Bostrom K, Watson KE, Horn S, Wortham C, Herman IM, Demer LL: Bone morphogenetic protein expression in human atherosclerotic lesions. J Clin Invest 1993, 91:1800-1809

7. Osman L, Chester AH, Sarathchandra P, Latif N, Meng W, Taylor PM Yacoub $\mathrm{MH}$ : A novel role of the sympatho-adrenergic system in regulating valve calcification. Circulation 2007, 116:I282-I287

8. Osman L, Yacoub MH, Latif N, Amrani M, Chester AH: Role of human valve interstitial cells in valve calcification and their response to atorvastatin. Circulation 2006, 114:1547-1552

9. Rajamannan NM, Subramaniam M, Rickard D, Stock SR, Donovan J, Springett M, Orszulak T, Fullerton DA, Tajik AJ, Bonow RO, Spelsberg $\mathrm{T}$ : Human aortic valve calcification is associated with an osteoblast phenotype. Circulation 2003, 107:2181-2184

10. Tanaka K, Sata M, Fukuda D, Suematsu Y, Motomura N, Takamoto S Hirata Y, Nagai R: Age-associated aortic stenosis in apolipoprotein E-deficient mice. J Am Coll Cardiol 2005, 46:134-141

11. Yip CY, Chen JH, Zhao R, Simmons CA: Calcification by valve interstitial cells is regulated by the stiffness of the extracellular matrix. Arterioscler Thromb Vasc Biol 2009, 29:936-942

12. Liu AC, Gotlieb Al: Transforming growth factor-beta regulates in vitro heart valve repair by activated valve interstitial cells. Am J Pathol 2008, 173:1275-1285

13. Liu AC, Joag VR, Gotlieb Al: The emerging role of valve interstitial cell phenotypes in regulating heart valve pathobiology. Am J Pathol 2007, 171:1407-1418

14. Sacks MS, Yoganathan AP: Heart valve function: a biomechanical perspective. Philos Trans R Soc Lond B Biol Sci 2007, 362:1369-1391

15. O'Brien KD: Pathogenesis of calcific aortic valve disease: a disease process comes of age (and a good deal more). Arterioscler Thromb Vasc Biol 2006, 26:1721-1728

16. Balachandran K, Sucosky P, Jo H, Yoganathan AP: Elevated cyclic stretch alters matrix remodeling in aortic valve cusps: implications for degenerative aortic valve disease? Am J Physiol Heart Circ Physiol 2009, 296:H756-H764

17. $\mathrm{Ku} \mathrm{CH}$, Johnson $\mathrm{PH}$, Batten $\mathrm{P}$, Sarathchandra $\mathrm{P}$, Chambers $\mathrm{RC}$ Taylor PM, Yacoub $\mathrm{MH}$, Chester $\mathrm{AH}$ : Collagen synthesis by mesen- chymal stem cells and aortic valve interstitial cells in response to mechanical stretch. Cardiovasc Res 2006, 71:548-556

18. Sucosky P, Padala M, Elhammali A, Balachandran K, Jo H, Yoganathan AP: Design of an ex vivo culture system to investigate the effects of shear stress on cardiovascular tissue. J Biomech Eng 2008 , 130:035001

19. Rabkin SW: The association of hypertension and aortic valve sclerosis. Blood Press 2005, 14:264-272

20. Thubrikar M: The aortic valve. Boca Raton, CRC Press, 1990

21. Metzler SA, Pregonero CA, Butcher JT, Burgess SC, Warnock JN: Cyclic strain regulates pro-inflammatory protein expression in porcine aortic valve endothelial cells. J Heart Valve Dis 2008, 17:571-577; discussion 578

22. Balachandran K, Konduri S, Sucosky P, Jo H, Yoganathan AP: An ex vivo study of the biological properties of porcine aortic valves in response to circumferential cyclic stretch. Ann Biomed Eng 2006, 34:1655-1665

23. Lomashvili KA, Cobbs S, Hennigar RA, Hardcastle KI, O'Neill WC: Phosphate-induced vascular calcification: role of pyrophosphate and osteopontin. J Am Soc Nephrol 2004, 15:1392-1401

24. Leskinen Y, Paana T, Saha H, Groundstroem K, Lehtimaki T, Kilpinen $\mathrm{S}$, Huhtala $\mathrm{H}$, Airaksinen J: Valvular calcification and its relationship to atherosclerosis in chronic kidney disease. J Heart Valve Dis 2009, 18:429-438

25. Merryman WD, Lukoff HD, Long RA, Engelmayr GC, Jr., Hopkins RA Sacks MS: Synergistic effects of cyclic tension and transforming growth factor-beta1 on the aortic valve myofibroblast. Cardiovasc Pathol 2007, 16:268-276

26. Zimmerman LB, De Jesus-Escobar JM, Harland RM: The Spemann organizer signal noggin binds and inactivates bone morphogenetic protein 4. Cell 1996, 86:599-606

27. Sorescu GP, Song H, Tressel SL, Hwang J, Dikalov S, Smith DA, Boyd NL, Platt MO, Lassegue B, Griendling KK, Jo H: Bone morphogenic protein 4 produced in endothelial cells by oscillatory shear stress induces monocyte adhesion by stimulating reactive oxygen species production from a nox1-based NADPH oxidase. Circ Res 2004, 95:773-779

28. Sucosky P, Balachandran K, Elhammali A, Jo H, Yoganathan AP Altered shear stress stimulates upregulation of endothelial VCAM-1 and ICAM-1 in a BMP-4- and TGF-beta1-dependent pathway. Arterioscler Thromb Vasc Biol 2009, 29:254-260

29. Simmons CA, Grant GR, Manduchi E, Davies PF: Spatial heterogeneity of endothelial phenotypes correlates with side-specific vulnerability to calcification in normal porcine aortic valves. Circ Res 2005 96:792-799

30. Mohler ER, 3rd, Chawla MK, Chang AW, Vyavahare N, Levy RJ Graham L, Gannon FH: Identification and characterization of calcifying valve cells from human and canine aortic valves. J Heart Valve Dis 1999, 8:254-260

31. Rajamannan NM, Sangiorgi G, Springett M, Arnold K, Mohacsi T, Spagnoli LG, Edwards WD, Tajik AJ, Schwartz RS: Experimental hypercholesterolemia induces apoptosis in the aortic valve. J Heart Valve Dis 2001, 10:371-374

32. Chang K, Weiss D, Suo J, Vega JD, Giddens D, Taylor WR, Jo H Bone morphogenic protein antagonists are coexpressed with bone morphogenic protein 4 in endothelial cells exposed to unstable flow in vitro in mouse aortas and in human coronary arteries: role of bone morphogenic protein antagonists in inflammation and atherosclerosis. Circulation 2007, 116:1258-1266

33. Platt MO, Xing Y, Jo H, Yoganathan AP: Cyclic pressure and shear stress regulate matrix metalloproteinases and cathepsin activity in porcine aortic valves. J Heart Valve Dis 2006, 15:622-629 